\title{
A Comparison of Neural Networks and Classical Discriminant Analysis in Predicting Students' Mathematics Placement Examination Scores
}

\author{
Stephen J. Sheel ${ }^{1}$, Deborah Vrooman ${ }^{2}$, R.S. Renner ${ }^{3}$, Shanda K. Dawsey ${ }^{4}$ \\ ${ }^{1}$ Department of Computer Science \\ Coastal Carolina University \\ Steves@coastal.edu \\ ${ }^{2}$ Department of Mathematics \\ Coastal Carolina University \\ Vroomand@coastal.edu \\ ${ }^{3}$ Department of Computer Science \\ California State University, Chico \\ Renner@ecst.csuchico.edu \\ ${ }^{4}$ Coastal Carolina University \\ AVX Corporation \\ Dawsey@sccoast.net
}

\begin{abstract}
Implementing a technique that is efficient yet accurate for college student placement into the appropriate mathematics course is important. Coastal Carolina University currently groups students into entry-level mathematics courses based upon their scores on a mathematics placement examination given to incoming freshmen. This paper examines alternative placement strategies. Using multiple regression analysis, the accumulative high school grade point average, mathematics SAT, and the final grade in Algebra II were found to be the best predictors of success on a mathematics placement examination. Entry-level mathematics placement based on neural networks and discriminant analysis is contrasted with placement results from the mathematics placement test. It is found that a neural network can outperform classical discriminant analysis in correctly predicting the recommended mathematics placement. Consequently, a trained neural network can be an effective alternative to a written mathematics placement test.
\end{abstract}

\section{Introduction}

As technology advances, methodologies evolve to enhance our abilities to perform arduous tasks more expediently. Using modern computer technologies not only makes completing tasks more efficient, but also may achieve a higher degree of accuracy. For instance, classifying students into an appropriate entry-level course is often a time-consuming task. The traditional classification method is to administer a placement exam to each student for the purpose of measuring his or her ability in a particular subject and/or area. Testing students requires time and energy. The exam 
questions must be developed and analyzed, and a process for administrating and scoring the exams must be implemented. In administering the exam, there is the arrangement of a time and place for taking the exam, production of a physical copy of the exam, and the delegation of a proctor. Once the exams are given, the exams must be scored and students, based upon their scores, placed into the appropriate entry-level course. Similarly, a computer-based test requires design, programming, implementation, and scoring. Several tools can be used for predictive purposes in applied research. In studies where the criterion variable is nominal rather than continuous, neural networks and classical discriminant analysis can be used to predict group membership.

Currently at Coastal Carolina University, most incoming freshmen and transfer students take a mathematics placement test prior to course enrollment. Students are then placed into a mathematics course according to their performance. The entry -level mathematics courses are as follows: Math 130I -- Intensive College Algebra, Math 130 -- College Algebra, Math 131 -- Trigonometry and Analytic Geometry, and Math 160 -- Calculus I. The placement test created by the Department of Mathematics and Statistics is being utilized by all colleges at Coastal Carolina University, except the E. Craig Wall College of Business. Students entering the College of Business are assigned to a mathematics class according to their academic achievement in secondary education. Prior to summer 2000, the mat hematics placement test was given in the form of a written instrument usually during a student orientation session in the summer before fall enrollment. In summer 2000, students applying to Coastal Carolina University had the option of taking a computer based mathematics placement test online over the Internet before arriving or taking it at the University.

Neural networks and discriminant analysis are two techniques that can be employed for membership classification. This study compares the effectiveness of placement based on neural networks and discriminant analysis with that of traditional testing. The results for both the neural network and discriminant analysis are compared to the results obtained by the mathematics placement test. For this study, results are contrasted for the mathematics placement test given before and after the advent of the computer based online testing. If a trained neural network or prediction equation based on discriminant analysis yields statistically similar results to the mathematics placement test, then one or the other could be used for entry-level placement in mathematics courses.

\section{Related Applications in Education}

Within the last decade, neural networks and discriminant analysis have been compared as predictors of success or failure in various disciplines. Gorr, Nagin, and Szczypula [5] conducted a comparative study of neural networks and statistical models for predicting college students'grade point averages. Discriminant analysis is included as one of the statistical models compared to a neural network. Their study concluded that the neural network serves as a better predictor than discriminant analysis. On the other hand, Wilson and Hardgrave [8] found that the discriminant analysis approach predicts graduate student success in a master's level business administration (MBA) program better than the neural network approach. 
The use of neural networks as a predictor has increased over the past few years. Cripps [3] uses a neural network to predict grade point averages of Middle Tennessee State University students. According to Carbone and Piras [2], neural networks are instrumental in predicting high school dropouts. In Nelson and Henriksen's study [6], a neural network uses input from student responses on a mathemat ics placement examination given to incoming students at Ball State University and outputs the mathematics course in which each student should be placed. The implementation of neural networks as a prediction tool continues to increase.

\section{Setting-up the Experiments}

When assigning college students to an entry-level mathematics course on the basis of their high school performance, certain factors may contribute to student performance in mathematics. High school grade point average (GPA), class rank, Scholastic Aptitude Tests (SAT), grades earned in high school algebra I and II, geometry, and advanced mathematics courses are academic performance indicators that have the potential of affecting mathematics course placement in college. Multiple correlation coefficients adjusted to beta weights were used to analyze the variance of these predictors of scores on the mathematics placement test. The three factors chosen to have the most influence on mathematics placement test scores are high school GPA, SAT mathematics score, and final grade in high school algebra II.

The scale used for high school GPA and the final grade in high school algebra II is 0.0 to 4.0 , with 0.0 representing the lowest possible score or an 'F", 1.0 representing a "D", 2.0 representing a "C", 3.0 representing a "B", and 4.0 representing a perfect score or an "A". The SAT mathematics score serves as a measurement of a student's overall mathematical ability. The scale for the SAT mathematics score ranges from a low of 200 to a high of 800 .

\section{Neural Network Approach}

An artificial neural network represents a connectionist structure whereby information is implicitly stored within the network connections and the solution is uncovered over a long series of computations. The neural network used in this study is a recurrent backpropagation network generated using a commercial simulation tool called BrainMaker Professional [1]. A three-layer feed-forward architecture was chosen: a three-unit input layer, ten-unit hidden layer, and a four-unit output layer. The first layer represents the inputs to the network (GPA, SAT, AlgII), and the output layer represents the classification (appropriate entry-level course). The hidden layers are purely computational units for the network.

Backpropagation is a supervised learning algorithm, which requires "training" the neural network. Training is performed using labeled data (data in which the target outputs are known). Values associated with network connections oscillate until the network stabilizes (reaches minimum error criteria), at which point the connection values (weights) are frozen (fixed). This fixed topology is then used to test the 
network against new unseen data representing student records that were not part of the training set.

In this experiment, the network is trained using data for entry-level college students grouped according to their performance on the mathematics placement test. The student's high school grade point average, SAT mathematics score, and high school algebra II score are used in the input layer. The output layer consists of four nodes representing courses in which the student may be placed into by the mathematics placement exam. A subset of the available data is held out for testing.

\section{Classical Approach (Statistical Modeling)}

Discriminant analysis is a statistical technique used to classify objects into distinct groups, based on a set of criteria or characteristics of the objects. Fisher's Linear Discriminant Analysis (FLDA) is a favorable classification rule that works well in situations where the groups to be discriminated are linearly separable. Objects are grouped according to their discriminant score, which is computed based on the object's observed values of the discriminating criteria or characteristics [4].

\section{Data Collection}

Academic information for students entering Coastal Carolina University from the fall semester of 1995 to the spring semester of 1997 was obtained through the Office of Institutional Advancement and used for the first data set. The second data set co nsists of students entering Coastal Carolina University in the fall of 2000 who took the computer based placement test. The data was divided into the following categories for each student: high school grade point average, high school rank, SAT verbal, SA T mathematics, algebra I, algebra II, geometry, advanced mathematics and mathematics placement examination scores. All student records with blank fields were deleted from the study. The first data set is composed of students who were correctly placed in an entry-level mathematics class using the traditional written instrument. Only records of students receiving a final grade of " $\mathrm{C}$ " or higher were selected. In the second data set, students with a final grade of " $\mathrm{D}$ " or higher were included in the analysis. The total number of records in the first data set is 458 and the second data set is 198 .

\section{$7 \quad$ Results and Conclusions}

Using the first data representing the written placement test, the 458 student records are randomly ordered and assigned to two equal data partitions. The first partition is used to train the neural network and to create the discriminant analysis predictive equation. The second partition is used to test the trained neural network and the predictive discriminant analysis equation. Both methodol ogies use the overall high school GPA, the SAT mathematics score, and the final grade in Algebra II as input, and the mathematics placement result as the dependent variable. 
The trained neural network correctly places 206 out of the 229 (90.0\%) records in the testing data set when compared to the mathematics placement test. The predictive equations derived using discriminant analysis correctly places 155 out of $229(67.7 \%)$ records in the testing data set. These experiments reflect a $22.2 \%$ positive difference in classification performance rate of the neural network and discriminant analysis. This difference represents an improvement of $68.9 \%$, where improvement is measured by the reduction in classification error (e.g. (74-23)/74), see Table 1, row 1).

Using a chi-square contingency test, the null hypothesis of independence between the placement results and the methodology used is rejected. The placement results reflect a 0.01 level dependency on methodology. Hence, there is not a significant difference between the mathematics placement test and the trained neural network. This insignificance implicates the neural network as a valid replacement candidate for the mathematics placement test, at $90 \%$ accuracy.

Table 1. Classification results for comparison methods

\begin{tabular}{|c|c|c|c|c|c|c|}
\hline & $\begin{array}{c}\# \text { Of } \\
\text { Records }\end{array}$ & $\begin{array}{c}\text { Nnet } \\
\text { Misclass. } \\
\text { Records }\end{array}$ & $\begin{array}{c}\text { Nnet } \\
\text { Class. } \\
\text { Rate }\end{array}$ & $\begin{array}{c}\text { D.A. } \\
\text { Misclass. } \\
\text { Records }\end{array}$ & $\begin{array}{c}\text { D.A. } \\
\text { Class. } \\
\text { Rate }\end{array}$ & $\begin{array}{c}\text { Improvement of } \\
\text { nnet over D.A. } \\
\text { (Misclassification } \\
\text { Reduction) }\end{array}$ \\
\hline $\begin{array}{c}\text { Experiment } \\
1\end{array}$ & 229 & 23 & $90.0 \%$ & 74 & $67.7 \%$ & $68.9 \%$ \\
\hline $\begin{array}{c}\text { Experiment } \\
2\end{array}$ & 99 & 27 & $72.7 \%$ & 25 & $74.7 \%$ & $-8.0 \%$ \\
\hline \hline Average & - & 50 & $81.4 \%$ & 99 & $71.2 \%$ & $49.5 \%$ \\
\hline
\end{tabular}

In a similar manner, a second neural network was created to analyze the student placement data for the students taking the online computer based mathematics placement test. The trained neural network correctly places 72 of the $99(72.7 \%)$ records in the testing data set. The predictive equations derived using discriminant analysis correctly places 74 of the 99 (74.7\%) records. These experiments reflect a $2.0 \%$ negative difference in classification performance rate of the neural network and discriminant analysis, or an $8.0 \%$ increase in classification error (see Table 1, row 2). In this experiment, the results from neither method appear to be significant, and the dependency on the mathematics placement test is more significant.

The network generated for the first experiment outperformed the discriminant analysis technique by a significant amount. The network generated for the second experiment slightly under-performed the discriminant analysis technique, but not by a significant amount. When averaging the two experiments the results fall clearly in favor of the neural network, with an average misclassification rate of $81.4 \%$, as compared to discriminant analysis with an average misclassification rate of $71.2 \%$. The average improvement in terms of record classification error reduction is $49.5 \%$ (see Table 1, row 3). Due to the degree of difference between training data set sizes, 
one may speculate that the network in the first experiment was able to benefit from a larger train ing set size. The training size and the train -test split within this problem domain will be issues left for future research.

The results from these two experiments show that trained neural networks can be used with a minimal list of input parameters as a tool to effectively place students into entry-level mathematics courses. Such networks demonstrate an ability to improve upon discriminant analysis techniques, as shown here by as much as $68.9 \%$. The neural network in the second data set performed as well as discriminant analysis, but was not statistically significant to the results of the actual mathematics placement test. When contrasted to the logistical difficulties of testing, scoring, and reporting the scores on a paper mathematics placement test, neural network methods also provide greater temporal efficiency and an opportunity for administrative cost reduction. Future work will explore the use of NNES ensemble networks [7], and alternative training methods to increase the performance of neural ne tworks.

\section{References}

1. BrainMaker Professional Users Guide and Reference Manual, $4^{\text {th }}$ Ed., California Scientific Software, Nevada City, CA (1993)

2. Carbone, V., Piras, G.: "Palomar project: Predicting school renouncing dropouts, using the artificial neural networks as a support for educational policy decisions", Substance Use \& Misuse, Vol.33, No.3 (1998) 717-750

3. Cripps, A.: "Predicting grade point average using clustering and ANNs", Proceedings of World Congress on Neural Networks [CD-ROM], 486-490. Available: INSPEC Abstract Number C9807-7110-003 (1996)

4. Guertin, W.H., Bailey, J.P.: Introduction to Modern FACTER ANALYSIS, Ann Arbor, MI: Edward Brothers, Inc. (1970)

5. Gorr, W. L., Nagin, D., Szczypula, J.: "Comparative study of artificial neural network and statistical models for predicting student grade point averages" International Journal of Forecasting, Vol.10, No.2 (1994) 17-34

6. Nelson, C.V., Henriksen, L.W.: 'Using neural net technology to enhance the efficiency of a computer adaptive testing application", Mid-Western Educational Research Association Annual Meeting [Microfiche], 1-40. Available: ERIC Number ED387122 (1994)

7. Renner, R.S., Lacher, R.C.: "Combining Constructive Neural Networks for Ensemble Classification," Proceedings of the Fifth Joint Conference on Information Sciences (2000) 887-891

8. Wilson, R.L., Hardgrave, B.C.: "Predicting graduate student success in an MBA program: Regression versus classification", Educational and Psychological Measurement, vol.55, No.2 (1995) 186-195 